\title{
SÍNTESE E REATIVIDADE DE COMPLEXOS PLATINA-TRIFENILESTIBINA: UMA REVISÃO BIBLIOGRÁFICA
}

Roberto Santos Barbiéri*

Faculdade de Minas, 36880-000 Muriaé - MG, Universidade Vale do Rio Verde, 37410-000 Três Corações - MG, Brasil Carlos Roberto Bellato

Departamento de Química, Universidade Federal de Viçosa, 36570-000 Viçosa - MG, Brasil

Antonio Carlos Massabni

Universidade Estadual Paulista "Julio de Mesquita Filho", CP 355, 14801-970 Araraquara - SP, Brasil

Recebido em 10/12/04; aceito em 2/9/05; publicado na web em 16/2/06

\begin{abstract}
SYNTHESIS AND REACTIVITY OF TRIPHENYLSTIBINE-PLATINUM COMPLEXES: A BIBLIOGRAPHIC REVISION. This article deals with synthesis and reactivity of complexes with triphenylstibine $\left(\mathrm{SbPh}_{3}\right)$ as the ligand. A comparative study of analogous complexes of triphenylphosphine $\left(\mathrm{PPh}_{3}\right)$ and triphenylarsine $\left(\mathrm{AsPh}_{3}\right)$ with platinum in the oxidation states zero, two and four is included. The bibliographic revision includes publications since 1936, when the first $\mathrm{Pt}(\mathrm{II})$ complex with triphenylstibine was described.
\end{abstract}

Keywords: platinum; triphenylstibine; complexes.

\section{INTRODUÇÃO}

Existe um interesse muito grande em relação aos complexos de platina contendo trifenilfosfina $\left(\mathrm{PPh}_{3}\right)$, enquanto que os complexos com trifenilarsina $\left(\mathrm{AsPh}_{3}\right)$ são bem menos estudados e é relativamente pequeno o número de trabalhos sobre compostos de platina com trifenilestibina $\left(\mathrm{SbPh}_{3}\right)$. Além disso, a maior parte dos artigos relativos a complexos de platina com $\mathrm{SbPh}_{3}$ foi produzida nos anos 70 e 80 do século passado.

Neste trabalho, é feita uma revisão bibliográfica sobre síntese e reatividade desta classe de compostos, que inclui alguns exemplos sintetizados pelos autores.

\section{COMPLEXOS DE PLATINA(II) COM TRIFENILESTIBINA}

A primeira referência a um composto de platina com $\mathrm{SbPh}_{3}$ foi feita por Jensen, em 1936, que preparou o $\left[\mathrm{PtCl}_{2}\left(\mathrm{SbPh}_{3}\right)_{2}\right]$ na reação de $\mathrm{K}_{2}\left[\mathrm{PtCl}_{4}\right]$ com $\mathrm{SbPh}_{3}$. Em seu artigo, Jensen descreveu a preparação de duas dezenas de compostos de platina(II) com fosfinas, arsinas e estibinas, que foram utilizadas em estudos de isomeria e medidas de momentos de dipolo ${ }^{1}$. A partir do $\left[\mathrm{PtCl}_{2}\left(\mathrm{SbPh}_{3}\right)_{2}\right]$ foram preparados os derivados $\left[\mathrm{Pt}(\mathrm{CNO})_{2} \mathrm{~L}_{2}\right]\left(\mathrm{L}=\mathrm{PBuPh}_{2}, \mathrm{PBu}_{2} \mathrm{Ph}\right.$, $\left.\mathrm{PBu}_{3}, \mathrm{SbPh}_{3}\right)^{2},\left[\mathrm{Pt}(\mathrm{NCO}) \mathrm{L}_{2}\right](\mathrm{L}=$ piridina, $\alpha$ - e $\beta$-picolina, bipiridina, $\left.\mathrm{AsPh}_{3}, \mathrm{SbPh}_{3}\right)^{3}$ e $\left[\mathrm{Pt}\left(\mathrm{NO}_{3}\right)_{2}\left(\mathrm{SbPh}_{3}\right)_{2}\right]^{4}$, empregados em estudos de condutometria, espectroscopias UV-visível e no infravermelho e da natureza de ligações sigma e pi.

A reação de $\left[\mathrm{PtPh}_{2}(\mathrm{dmso})_{2}\right]$, preparado a partir de $\mathrm{K}_{2}\left[\mathrm{PtCl}_{4}\right]$ e $\mathrm{SnMe}_{3} \mathrm{Ph}$ em dimetilsulfóxido (dmso), com diversos ligantes possibilitou a obtenção de complexos do tipo $\left[\mathrm{PtPh}_{2} \mathrm{~L}_{2}\right](\mathrm{L}=$ difenilfosfinoetano, bipiridina, $\left.\mathrm{PEt}_{3}, \mathrm{PMePh}_{2}, \mathrm{PPh}_{3}, \mathrm{AsPh}_{3}, \mathrm{SbPh}_{3}\right)^{5}$.

Hulley et $a .^{6}{ }^{6}$, em 1970, estudaram algumas reações do complexo $\mathrm{H}\left[\mathrm{PtCl}(\mathrm{acac})_{2}\right]$ com vários ligantes e obtiveram complexos do tipo $[\mathrm{PtCl}(\mathrm{acac}) \mathrm{L}]\left(\mathrm{acac}=\right.$ acetilacetonato; $\mathrm{L}=\mathrm{PPh}_{3}, \mathrm{AsPh}_{3}$, $\mathrm{PEtPh}_{2}, \mathrm{PEt}_{2} \mathrm{Ph}, \mathrm{PEt}_{3}, \mathrm{P}\left(\mathrm{C}_{6} \mathrm{H}_{11}\right)_{3}, \mathrm{P}(\mathrm{OPh})_{3}, \mathrm{P}(\mathrm{OMe})_{3}$, piridina, $\mathrm{C}_{2} \mathrm{H}_{4}$,

*e-mail: barbieri@faminas.edu.br
$\mathrm{C}_{3} \mathrm{H}_{6}$, cis- e trans- $\mathrm{C}_{4} \mathrm{H}_{8}, 2,3$-dimetil-2-buteno, $\mathrm{PhC} \equiv \mathrm{CPh}, \mathrm{SbPh}_{3}$ ). Clark e colaboradores ${ }^{7}$ estudaram o efeito trans em complexos do tipo $[\mathrm{PtCl}(\mathrm{P}-\mathrm{C}) \mathrm{L}]\left(\mathrm{P}-\mathrm{C}={ }^{t} \mathrm{Bu}_{2}-\mathrm{PCMe}_{2} \mathrm{CH}_{2}-; \mathrm{L}=\mathrm{PEt}_{3}, \mathrm{PPr}_{3}\right.$, $\mathrm{P}\left(\mathrm{C}_{6} \mathrm{H}_{11}\right)_{3}, \mathrm{PMe}^{t} \mathrm{Bu}_{3}, \mathrm{P}^{t} \mathrm{Bu}_{3}, \mathrm{PMePh}_{2}, \mathrm{PPh}_{3}, \mathrm{P}^{t} \mathrm{BuPh}_{2}, \mathrm{P}(\mathrm{OPh})_{3}, \mathrm{AsPh}_{3}$, $\mathrm{CO}$, piridina, $\mathrm{Me}_{2} \mathrm{~S}, \mathrm{SbPh}_{3}$ ), os quais foram obtidos a partir do complexo dímero $[\mathrm{PtCl}(\mathrm{P}-\mathrm{C})]_{2}$ em reação com o ligante, em meio benzênico. Observou-se que a reação do dímero com $\mathrm{AsPh}_{3}$ levava à formação de trans-[PtCl$\left.\left(\mathrm{AsPh}_{3}\right)(\mathrm{P}-\mathrm{C})\right]$, enquanto que na reação com $\mathrm{SbPh}_{3}$ formavam-se cis- e trans- $\left[\mathrm{PtCl}(\mathrm{P}-\mathrm{C})\left(\mathrm{SbPh}_{3}\right)\right]^{7}$. Os complexos com $\mathrm{SbPh}_{3}$ tratados com $\mathrm{NaBH}_{4}$ e $\mathrm{PBu}_{3}$ produziam o complexo binuclear $\left[\mathrm{Pt}(\mathrm{H})(\mu-\mathrm{H})\left(\mathrm{PBu}_{3}\right)\right]_{2}{ }^{8}$. A partir de $[\mathrm{PtCl}(\mathrm{P}-\mathrm{C}) \mathrm{L}]$ tratado com excesso de $\mathrm{SnCl}_{2}$, em diclorometano, Goel e Goel ${ }^{9}$ obtiveram $\left[\mathrm{Pt}\left(\mathrm{SnCl}_{3}\right)(\mathrm{P}-\mathrm{C}) \mathrm{L}\right]\left(\mathrm{L}=\mathrm{AsPh}_{3}, \mathrm{CO}, \mathrm{P}\left(\mathrm{C}_{6} \mathrm{H}_{11}\right)_{3}, \mathrm{P}^{t} \mathrm{Bu}_{2} \mathrm{Me}\right.$, $\mathrm{PPh}_{2} \mathrm{Me}, \mathrm{PPh}_{3}, \mathrm{P}\left(p-\mathrm{ClC}_{6} \mathrm{H}_{4}\right)_{3}, \mathrm{P}\left(p-\mathrm{FC}_{6} \mathrm{H}_{4}\right)_{3}, \mathrm{P}\left(p-\mathrm{MeC}_{6} \mathrm{H}_{4}\right)_{3}, \mathrm{P}(p-$ $\left.\mathrm{MeOC}_{6} \mathrm{H}_{4}\right)_{3}, \mathrm{SbPh}_{3}$. Dados espectroscópicos de ${ }^{31} \mathrm{P}-\mathrm{RMN}$ indicaram que o acoplamento $\mathrm{J}_{\mathrm{Pt}-\mathrm{P}}$ nesses complexos aumenta na série $\mathrm{P}$ $>\mathrm{As}>\mathrm{Sb}$, que corresponde às ordens de basicidade e de influência trans dos ligantes?.

$\mathrm{O}$ tratamento de $\left[\mathrm{PtCl}_{2} \mathrm{~L}_{2}\right]\left(\mathrm{L}=\mathrm{PPh}_{3}, \mathrm{AsPh}_{3}, \mathrm{SbPh}_{3}\right)$ com $\mathrm{Ag}\left[\mathrm{BF}_{4}\right]$ produz $\left[\mathrm{PtCl}_{2} \mathrm{~L}_{4}\right]\left[\mathrm{BF}_{4}\right]_{2}$, o qual reage com monóxido de carbono para formar $\left[\mathrm{PtCl}(\mathrm{CO}) \mathrm{L}_{2}\right]\left[\mathrm{BF}_{4}\right]$. Se L $=\mathrm{AsPh}_{3}$, o complexo carbonílico reage com metanol ou com etanol formando $\left[\mathrm{PtCl}\left(\mathrm{AsPh}_{3}\right)(\mathrm{COOR})\right](\mathrm{R}=\mathrm{Me}, \mathrm{Et})$; quando $\mathrm{L}=\mathrm{SbPh}_{3}$, não ocorre reação ${ }^{10}$.

$\left[\mathrm{NBu}_{4}\right]_{2}\left[\mathrm{Pt}(\mu-\mathrm{Cl})\left(\mathrm{C}_{6} \mathrm{Cl}_{5}\right)_{2}\right]_{2}$, sob refluxo em etanol com excesso de $\mathrm{SbPh}_{3}$, forma cis- $\left[\mathrm{Pt}\left(\mathrm{C}_{6} \mathrm{Cl}_{5}\right)_{2}\left(\mathrm{SbPh}_{3}\right)_{2}\right]$ e, sob agitação em acetona, juntamente com quantidade estequiométrica de trifenilestibina, produz $\left[\mathrm{NBu}_{4}\right]$ cis- $\left[\mathrm{PtCl}\left(\mathrm{C}_{6} \mathrm{Cl}_{5}\right)_{2}\left(\mathrm{SbPh}_{3}\right)\right]^{11}$. O complexo de platina contendo tetraidrotiofeno (tht) coordenado $\left[\mathrm{NBu}_{4}\right]\left[\mathrm{PtPh}_{3} \mathrm{~L}\right]\left(\mathrm{L}=\mathrm{PPh}_{3}\right.$, $\left.\mathrm{AsPh}_{3}, \mathrm{SbPh}_{3}\right)^{11}$. $\left[\mathrm{NBu}_{4}\right]_{2}$-trans- $\left[\mathrm{PtCl}_{2}\left(\mathrm{C}_{6} \mathrm{~F}_{5}\right)_{2}\right]$ tratado com nitrato ou clorato de prata gera $\left[\mathrm{NBu}_{4}\right]_{2}\left[\mathrm{Ag}_{2} \mathrm{Pt}_{2} \mathrm{Cl}_{4}\left(\mathrm{C}_{6} \mathrm{~F}_{5}\right)_{4}\right]$, um cluster tetranuclear que contém ligações $\mathrm{Pt}-\mathrm{Ag}$ e $\mathrm{Ag}-\mathrm{Ag}$. $\mathrm{O}$ cluster reage com $\mathrm{AsPh}_{3}$ ou $\mathrm{SbPh}_{3}$, provocando precipitação de cloreto de prata e formação de uma mistura de trans- $\left[\mathrm{Pt}\left(\mathrm{C}_{6} \mathrm{~F}_{5}\right)_{2} \mathrm{~L}_{2}\right]$ e $\left[\mathrm{NBu}_{4}\right]_{2}$-trans$\left[\mathrm{PtCl}_{2}\left(\mathrm{C}_{6} \mathrm{~F}_{5}\right)_{2}\right]^{11}$. Da reação de $\left[\mathrm{Pt}\left(\mathrm{C}_{6} \mathrm{~F}_{5}\right)_{2}\right]$ em tetraidrofurano com ligantes adequados, obtém-se $\left[\mathrm{Pt}\left(\mathrm{C}_{6} \mathrm{~F}_{5}\right)_{2} \mathrm{~L}_{2}\right]\left(\mathrm{L}=\mathrm{NH}_{3}, \mathrm{PPh}_{3}, \mathrm{OPPh}_{3}\right.$, $\left.\mathrm{AsPh}_{3}, \mathrm{OAsPh}_{3}, \mathrm{SbPh}_{3}\right)^{12}$. 
Eaborn et al..$^{13}$, em 1976, e Eaborn et al..$^{13}$, em 1978, estudaram a reatividade de compostos diméricos dos tipos $\left[\mathrm{PtCl}_{2} \mathrm{R}_{2} \mathrm{Cl}_{2}\right]$ e $\left[\mathrm{Pt}_{2} \mathrm{Cl}_{2}(\mathrm{COR})_{2} \mathrm{~L}_{2}\right]\left(\mathrm{R}=\mathrm{Ph}, p-\mathrm{MeC}_{6} \mathrm{H}_{4}, p-\mathrm{Bu}^{\mathrm{t}} \mathrm{C}_{6} \mathrm{H}_{4}, p-\mathrm{Bu}^{\mathrm{t}} \mathrm{C}_{6} \mathrm{H}_{4} \mathrm{CO}, p-\right.$ $\mathrm{MeC}_{6} \mathrm{H}_{4} \mathrm{CO} ; \mathrm{L}=\mathrm{PEt}_{3}, \mathrm{PEt}_{2} \mathrm{Ph}, \mathrm{PMe}_{2} \mathrm{Ph}$ ) com diversos ligantes e prepararam o $\left[\mathrm{PtCl}\left(p-\mathrm{MeC}_{6} \mathrm{H}_{4}\right)\left(\mathrm{PEt}_{3}\right)\left(\mathrm{SbPh}_{3}\right)\right]$, caracterizado por ${ }^{31} \mathrm{P}-$ RMN. $\left[\mathrm{PtCl}_{2}\left(\mathrm{PPh}_{3}\right)_{4}\right]\left[\mathrm{BF}_{4}\right]$ reage com $\mathrm{SbPh}_{3}$ em nitrometano, para formar cis-[ $\left.\mathrm{PtCl}\left(\mathrm{PPh}_{3}\right)_{2}\left(\mathrm{SbPh}_{3}\right)\right]$.

Toniolo et al. ${ }^{14}$, em 1969, prepararam o $\left[\mathrm{Pt}(\mathrm{H})\left(\mathrm{PEt}_{3}\right)_{2} \mathrm{~L}_{\mathrm{ClO}}\right.$ $\left(\mathrm{L}=\mathrm{PPh}_{3}, \mathrm{AsPh}_{3}, \mathrm{P}\left(\mathrm{C}_{6} \mathrm{H}_{11}\right)_{3}, \mathrm{CO}, \mathrm{SbPh}_{3}\right)$. Miyamoto e Sasaky ${ }^{15}, \mathrm{em}$ 1976, obtiveram o trans- $\left[\mathrm{Pt}(\mathrm{H})\left\{\mathrm{P}\left(\mathrm{CH}_{2} \mathrm{Ph}\right)_{3}\right\}_{2} \mathrm{~L}\right] \mathrm{Z}\left(\mathrm{L}=\mathrm{NH}_{3}, \mathrm{PPh}_{3}\right.$, $\mathrm{AsPh}_{3}, \mathrm{SbPh}_{3} ; \mathrm{Z}=\mathrm{BPh}_{4}, \mathrm{ClO}_{4}$, tosilato), e Kato e colaboradores ${ }^{16}$, em 1976, prepararam o $[\mathrm{PtCl}(\mathrm{Y}) \mathrm{L}]\left[\mathrm{BPh}_{4}\right] \quad(\mathrm{Y}=$ $\mathrm{Ph}_{2}\left(\mathrm{CH}_{2}\right)_{\mathrm{n}} \mathrm{PPh}_{2} \mathrm{CHCOPh}, \mathrm{n}=1,2 ; \mathrm{L}=4$-metilpriridina, 3,5dimetilpirazol, $\left.\mathrm{PPh}_{3}, \mathrm{P}\left(\mathrm{C}_{6} \mathrm{H}_{11}\right)_{3}, \mathrm{PMePh}_{2}, \mathrm{P}(\mathrm{OMe})_{3}, \mathrm{AsPh}_{3}, \mathrm{SbPh}_{3}\right)$.

Alinson et al. ${ }^{17}$, em 1971 , estudaram a reatividade do $\left[\mathrm{Pt}\left(\mathrm{S}_{2} \mathrm{PPh}_{2}\right)_{2}\right]$, no qual o $\mathrm{S}_{2} \mathrm{PPh}_{2}$ se liga ao metal pelos dois átomos de enxofre, com diversos ligantes em benzeno. Verificaram que para $\mathrm{L}=\mathrm{PPh}_{3}, \mathrm{PMePh}_{2}$ e $\mathrm{PMe}{ }_{2} \mathrm{Ph}$ os produtos eram do tipo $\left[\mathrm{PtL}\left(\mathrm{S}_{2} \mathrm{PPh}_{2}\right)_{2}\right]$, onde um dos ligantes sulfurado está coordenado apenas por um dos átomos de enxofre, ou do tipo $\left[\mathrm{PtL}_{2}\left(\mathrm{~S}_{2} \mathrm{PPh}_{2}\right)\right]\left(\mathrm{S}_{2} \mathrm{PPh}_{2}\right)$. No caso de $\mathrm{L}=\mathrm{AsPh}_{3}$ e $\mathrm{SbPh}_{3}$ formavam-se apenas complexos do primeiro tipo.

Da reação de $\left.\mathrm{PtI}_{2}\left(\mathrm{C}_{8} \mathrm{H}_{8}\right)\right]\left(\mathrm{C}_{8} \mathrm{H}_{8}=1,3,5,7\right.$-ciclooctatetraeno $)$ com $\mathrm{SbPh}_{3}$, Kistner et al. ${ }^{18}$, em 1969, obtiveram o $\left[\mathrm{PtI}_{2}\left(\mathrm{SbPh}_{3}\right)_{2}\right]$ e, com $\mathrm{RMgI}\left(\mathrm{R}=\mathrm{Me}, \mathrm{Ph}, o-\mathrm{MeC}_{6} \mathrm{H}_{4}, m-\mathrm{MeC}_{6} \mathrm{H}_{4}, p-\mathrm{MeC}_{6} \mathrm{H}_{4}\right)$ prepararam $\left[\mathrm{R} 2 \mathrm{Pt}\left(\mathrm{C}_{8} \mathrm{H}_{8}\right) \mathrm{PtR}_{2}\right]$ ou $\left[\mathrm{PtR}_{2}\left(\mathrm{C}_{8} \mathrm{H}_{8}\right)\right]$ que, tratados com L $=\mathrm{PPh}_{3}, \mathrm{AsPh}_{3}$ e $\mathrm{SbPh}_{3}$ produziram $\left[\mathrm{PtR}_{2} \mathrm{~L}_{2}\right]$. A estabilidade térmica dos complexos do tipo $\left[\mathrm{PtR}_{2} \mathrm{~L}_{2}\right]$ cresce na ordem $\mathrm{P}>\mathrm{As}>\mathrm{Sb}$, com exceção do $\left[\mathrm{Pt}\left(o-\mathrm{MeC}_{6} \mathrm{H}_{4}\right)_{2}\left(\mathrm{SbPh}_{3}\right)_{2}\right]$, que decompõe em temperatura inferior à de decomposição dos análogos com $\mathrm{AsPh}_{3} \mathrm{e} \mathrm{PPh}_{3}$, devido à combinação de fatores estéricos do tamanho do $\mathrm{SbPh}_{3}$ e do grupo metila em posição orto no grupo $o$-tolila.

Complexos perfluoroalquilados de platina(II) foram preparados a partir da reação de $\left[\mathrm{PtMe}_{2}(\operatorname{cod})\right](\operatorname{cod}=1,5$-ciclooctadieno $)$ com $\mathrm{CF}_{3} \mathrm{I}$. Assim, foram obtidos compostos do tipo $\left[\mathrm{Pt}\left(\mathrm{CF}_{3}\right)_{2}(\mathrm{cod})\right]$ que, por reação com $\mathrm{L}=\mathrm{MeCN}, \mathrm{PMe}_{2} \mathrm{Ph}, \mathrm{AsMe}_{3}$, bipiridina, $\gamma$ picolina, $\mathrm{AsPh}_{3}, \mathrm{PhC} \equiv \mathrm{CPh}, \mathrm{Me}_{2} \mathrm{~N}\left(\mathrm{CH}_{2}\right)_{2} \mathrm{NMe}_{2}$ e $\mathrm{SbPh}_{3}$, produziram cis- $\left[\mathrm{Pt}\left(\mathrm{CF}_{3}\right)_{2} \mathrm{~L}_{2}\right]^{19}$.

Payne e Sthephan ${ }^{20}$, em 1981, prepararam e estudaram por ${ }^{31} \mathrm{P}-$ RMN complexos de platina com fosfinas quirálicas bidentadas, do tipo $\left[\mathrm{Pt}\left(\mathrm{CH}_{3}\right)\right.$ (quelante $\left.) \mathrm{L}\right] \mathrm{ClO}_{4}$ \{quelante $=\mathrm{RNPPh}_{2} ; \mathrm{L}=$ acetona, $\left.p-\mathrm{YC}_{5} \mathrm{H}_{4} \mathrm{~N}\left(\mathrm{Y}=\mathrm{Me}, \mathrm{Et}, \mathrm{CHO}, \mathrm{CO}_{2} \mathrm{Me}, \mathrm{H} \mathrm{e} \mathrm{NMe}\right)_{2}\right), \mathrm{PEt}_{3}, \mathrm{PPr}_{3}$, $\mathrm{P}\left(\mathrm{C}_{8} \mathrm{H}_{17}\right), \mathrm{PMePh}_{2}, \mathrm{PMe}_{2} \mathrm{Ph}, \mathrm{PPh}_{3}, \mathrm{P}\left(\mathrm{C}_{6} \mathrm{H}_{11}\right) \mathrm{Ph}_{2}, \mathrm{P}\left(\mathrm{C}_{6} \mathrm{H}_{11}\right) \mathrm{Ph}$, $\mathrm{P}\left(\mathrm{C}_{6} \mathrm{H}_{11}\right)_{3}, \mathrm{PPh}_{2} \mathrm{NEt}_{2}, \mathrm{AsPh}_{3}$ e $\left.\mathrm{SbPh}_{3}\right)$ \}.

Compostos do tipo $\left[\mathrm{Pt}(\mathrm{X})(\mathrm{Y}) \mathrm{L}_{2}\right]\left(\mathrm{X}, \mathrm{Y}=\mathrm{Cl}, \mathrm{Br}, \mathrm{I}, \mathrm{H}, \mathrm{Me}, \mathrm{C}(\mathrm{CN})_{3}\right.$, $\mathrm{NCO}, \mathrm{SCN}, \mathrm{N}_{2}, \mathrm{CN},(\mathrm{NC})_{2} \mathrm{C}=\mathrm{C}(\mathrm{CN})_{2} ; \mathrm{L}=\mathrm{PPh}_{3}, \mathrm{SbPh}_{3}$ ) foram estudados por espectroscopia fotoeletrônica de raios-X, tendo sido verificado que nos complexos $\left[\mathrm{PtX}_{2}\left(\mathrm{SbPh}_{3}\right)_{2}\right](\mathrm{X}=\mathrm{Cl}, \mathrm{NCO}, \mathrm{NCS})$ as energias de ligação no nível eletrônico $\operatorname{Pt}(4 \mathrm{f})$ são menores quando comparadas aos correspondentes compostos com $\mathrm{PPh}_{3}{ }^{21}$. Grutsch et al. ${ }^{22}$, em 1973, fizeram medidas de energias de ligação de elétrons nos níveis $\mathrm{Sn}(4 \mathrm{~d})$ e $3 \mathrm{~d}_{(5 / 2)}$ em uma série de compostos de estanho e, entre eles, o $\left[\mathrm{PtCl}\left(\mathrm{SnCl}_{3}\right)\left(\mathrm{SbPh}_{3}\right)_{2}\right]$. Para $\left[\mathrm{PtCl}\left(\mathrm{SnCl}_{3}\right) \mathrm{L}_{2}\right]\left(\mathrm{L}=\mathrm{PPh}_{3}\right.$, $\mathrm{AsPh}_{3}, \mathrm{SbPh}_{3}$ ), os valores dessas energias não apresentaram diferenças significativas.

Gavrilova e colaboradores ${ }^{23}$, em 1971, estudaram os espectros vibracionais na região do infravermelho para os compostos $\left[\mathrm{Pt}(\mathrm{H})(\mathrm{X})\left(\mathrm{PPh}_{3}\right)_{2}\right]$ e $\left[\mathrm{Pt}(\mathrm{H})\left(\mathrm{PPh}_{3}\right)_{2} \mathrm{~L}\right] \mathrm{ClO}_{4}\left(\mathrm{X}=\mathrm{Cl}, \mathrm{ClO}_{4} ; \mathrm{L}=\mathrm{PPh}_{3}\right.$, $\mathrm{C}_{2} \mathrm{H}_{4}, \mathrm{C}_{3} \mathrm{H}_{6}, \mathrm{CO}, \mathrm{NH}_{3}, \mathrm{MeNH}_{2}, \mathrm{EtNH}_{2}, \mathrm{NHMe}_{2}$, piridina, tiouréia) e verificaram que o estiramento Pt-N diminui com o aumento da influência trans do ligante trans à ligação $\mathrm{Pt}-\mathrm{H}$. A análise da série trans- $\left[\mathrm{Pt}(\mathrm{H})\left(\mathrm{PPh}_{3}\right) \mathrm{L}\right] \mathrm{ClO}_{4}\left(\mathrm{~L}=\mathrm{C}_{2} \mathrm{H}_{4}, \mathrm{C}_{3} \mathrm{H}_{6}, \mathrm{CO}, \mathrm{PPh}_{3}\right.$, piridina, $\left.\mathrm{SbPh}_{3}\right)$ obtida a partir do trans- $\left[\mathrm{Pt}(\mathrm{H})(\mathrm{X})\left(\mathrm{PPh}_{3}\right)_{2}\right]\left(\mathrm{X}=\mathrm{Br}, \mathrm{I}, \mathrm{NO}_{3}\right.$, $\mathrm{ClO}_{4}$ ) permitiu determinar a série de influência trans com base em dados de infravermelho, tendo sido comprovado que a influência trans do ligante $\mathrm{PPh}_{3}$ é praticamente igual à do $\mathrm{SbPh}_{3}^{23}$. Janos e Goggin $^{24}$, em 1984, estudaram setenta complexos do tipo cis$\left[\mathrm{PtX}_{2} \mathrm{~L}_{2}\right]$ e, com base nas frequiências de estiramento Pt-C, estabeleceram a série espectroquímica de influência trans $\mathrm{PPh}_{3}>\mathrm{SbPh}_{3}$ $>\mathrm{AsPh}_{3}$. A partir de medidas de $\mathrm{pKa}$, Gavrilova e colaboradores ${ }^{23}$ determinaram que complexos do tipo trans- $\left[\mathrm{PtCl}\left(\mathrm{NH}_{3}\right)_{2} \mathrm{~L} \mathrm{NO}_{3}\right.$ $\left(\mathrm{L}=\mathrm{PPh}_{3}, \mathrm{SbPh}_{3}\right)$ o efeito trans para o $\mathrm{PPh}_{3}$ é maior que para o $\mathrm{SbPh}_{3}$, implicando que os dois ligantes possuem grande habilidade como doador sigma e receptor pi, sendo que ambos efeitos são mais pronunciados para o ligante $\mathrm{PPh}_{3}$.

McAuliffe et al..$^{25}$, em 1977, prepararam uma série de compostos de paládio e platina com diversas estibinas. Os complexos $\left[\mathrm{MX}_{2}\left(\mathrm{SbR}_{3}\right)_{2}\right]\left(\mathrm{M}=\mathrm{Pd}, \mathrm{Pt} ; \mathrm{X}=\mathrm{Cl}, \mathrm{Br}, \mathrm{I}, \mathrm{SCN}, \mathrm{NO}_{2} ; \mathrm{R}=\mathrm{Et}, \mathrm{C}_{6} \mathrm{H}_{11}\right.$, $\mathrm{Ph}, o$-tolil, $m$-tolil, $p$-tolil) com ligantes mais volumosos como $\mathrm{Sb}\left(\mathrm{C}_{6} \mathrm{H}_{11}\right)_{3}$ e $\mathrm{Sb}(o \text {-tolil })_{3}$ apresentam-se na forma trans e os demais, com ligantes menos volumosos, geralmente possuem a forma cis. Os complexos foram caracterizados por análise elementar e espectroscopia UV-visível e infravermelho, o que possibilitou o estabelecimento da série de influência trans para os ligantes. No caso do complexo de platina, $\mathrm{SbPh}_{3}$ e tiocianato, em solução, deve haver um rearranjo como indicado na Equação a seguir:

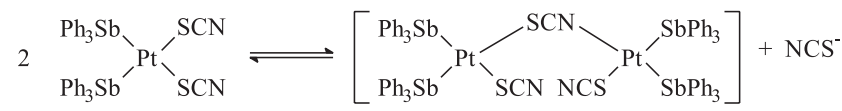

Appleton e colaboradores ${ }^{26}$, em 1972, estudaram por RMN uma série de compostos do tipo trans $\left[\mathrm{Pt}\left(\mathrm{CF}_{3}\right)\left(\mathrm{PMe}_{2} \mathrm{Ph}\right)_{2} \mathrm{~L}\right]\left[\mathrm{PF}_{6}\right](\mathrm{L}=$ $\mathrm{NCCH}=\mathrm{CH}_{2}, p-\mathrm{NCC}_{6} \mathrm{H}_{4} \mathrm{OMe}, \mathrm{HN}=\mathrm{C}(\mathrm{OMe}) \mathrm{C}_{6} \mathrm{~F}_{5}$, piridina, $p$ $\{\mathrm{NH}=\mathrm{C}(\mathrm{OMe})\}_{2} \mathrm{C}_{6} \mathrm{~F}_{4}, \mathrm{CO}, \mathrm{EtNC}, p-\mathrm{CNC}_{6} \mathrm{H}_{4} \mathrm{OMe}, \mathrm{PPh}_{3}, \mathrm{AsPh}_{3}$, $\mathrm{SbPh}_{3}$ ), tendo sido observada uma correlação linear entre $\mathrm{J}_{\mathrm{Pt}_{-\mathrm{CF}}} \mathrm{e}$ $\mathrm{J}_{\mathrm{Pt-Me}}$, exceto para os complexos com $\mathrm{SbPh}_{3}$ e CO. A discrepância observada nessa relação foi explicada em termos do efeito sinérgico das ligações Pt-Sb e Pt-CO. No entanto, o estudo dos complexos vinílicos trans-[PtXR $\left.\left(\mathrm{PMe}_{2} \mathrm{Ph}\right)_{2}\right]$ e trans-[PtXR $\left.\left(\mathrm{PMe}_{2} \mathrm{Ph}\right)_{2} \mathrm{~L}\right]\left[\mathrm{PF}_{6}\right]$, onde $\mathrm{X}=\mathrm{NCS}, \mathrm{NO}_{2}, \mathrm{CN}, \mathrm{EtNC}, \mathrm{CO}, \mathrm{AsPh}_{3}, \mathrm{SbPh}_{3}$, e R = $-\mathrm{C}\left(\mathrm{CF}_{3}\right)=\mathrm{C}(\mathrm{H}) \mathrm{OMe}$, também realizados por Appleton ${ }^{27}$, indicaram que os acoplamentos ${ }^{3} \mathrm{~J}_{\mathrm{Pt}-\mathrm{C}=\mathrm{C}-\mathrm{H}} \mathrm{e}^{3} \mathrm{~J}_{\mathrm{Pt}-\mathrm{C}-\mathrm{CF}_{3}}$ estavam linearmente correlacionados com o acoplamento ${ }^{2} \mathrm{~J}_{\mathrm{Pt}-\mathrm{Me}}$, exceto para o complexo com $\mathrm{SbPh}_{3}$, não tendo sido observada discrepância para o complexo com monóxido de carbono. Assim, efeitos sinérgicos não devem ser responsáveis pelos desvios observados e o valor do acoplamento ${ }^{2} \mathrm{~J}_{\mathrm{Pt}-\mathrm{Me}}$ é "anômalo por alguma razão desconhecida".

Da reação da base de Schiff marcada com ${ }^{15} \mathrm{~N}, o-\mathrm{HOC}_{6} \mathrm{H}_{4} \mathrm{CH}$ $=\mathrm{NCH}_{2} \mathrm{CH}_{2} \mathrm{OH}$ com $\mathrm{K}_{2}\left[\mathrm{PtCl}_{4}\right]$, em proporção estequiométrica do ligante $\mathrm{L}\left(\mathrm{L}=\mathrm{PBu}_{3}, \mathrm{P}(\mathrm{OEt})_{3}, \mathrm{P}(p-\mathrm{MePh})_{3}, \mathrm{AsBu}_{3}, \mathrm{As}(p-\mathrm{MePh})_{3}\right.$, dimetilsulfóxido, piperidina, piridina, $\mathrm{PhC} \equiv \mathrm{C}-, \mathrm{CH}_{3}\left(\mathrm{CH}_{2}\right)_{5} \mathrm{NH}_{2}$, $\mathrm{SbPh}_{3}$ ), foram preparados complexos esquematizados na Equação a seguir:

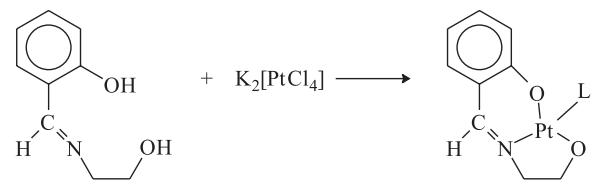

Estudos de RMN desses complexos permitiram estabelecer as constantes de acoplamento ${ }^{1} \mathrm{~J}_{\mathrm{Pt}, \mathrm{N}}$ e os deslocamentos $\delta^{195}$ e $\delta^{15}{ }_{\mathrm{N}}$, exceto para o complexo com $\mathrm{L}=\mathrm{SbPh}_{3}$ que foi observado apenas o deslocamento $\delta_{\mathrm{N}^{28}}^{15}$.

A série de complexos $[\mathrm{Pt}(\mathrm{Me})(\mathrm{dppe}) \mathrm{L}]\left[\mathrm{BF}_{4}\right](\mathrm{dppe}=$ difenilfosfinoetano; $\mathrm{L}=\mathrm{MeCN}$, piridina, $\left.\mathrm{CO}, \mathrm{P}(\mathrm{OPh})_{3}, \mathrm{PPh}_{3}, \mathrm{AsPh}_{3}, \mathrm{SbPh}_{3}\right)$ 
também foi estudada por RMN, para o estabelecimento da série de influência trans ${ }^{29}$. No caso do complexo com $\mathrm{L}=\mathrm{SbPh}_{3}$, o acoplamento ${ }^{2} \mathrm{~J}_{\mathrm{Pt}_{\mathrm{CH}}}$ não foi observado, sendo esse comportamento explicado em termos do acoplamento escalar entre os núcleos ${ }^{195} \mathrm{Pt}$ e os dois núcleos quadrupolares do antimônio $\left({ }^{121} \mathrm{Sb}, \mathrm{I}=5 / 2\right.$ e ${ }^{123} \mathrm{Sb}$, $\mathrm{I}=7 / 2)$ e da relaxação do núcleo de platina decorrente dessa interação ${ }^{29}$.

Medidas de espectroscopia Mössbauer do núcleo ${ }^{121} \mathrm{Sb}$ em complexos quadrado-planos do tipo $\left[\mathrm{MX}_{2} \mathrm{~L}_{2}\right]\left(\mathrm{M}=\mathrm{Pd}, \mathrm{Pt} ; \mathrm{L}=\mathrm{Cl}, \mathrm{I}_{2} \mathrm{NO}_{2}\right.$; $\left.\mathrm{L}=\mathrm{SbPh}_{3}, \mathrm{SbEt}_{3}, \mathrm{Sb}\left(\mathrm{C}_{6} \mathrm{H}_{11}\right)_{3}, \mathrm{Sb}\left(p-\mathrm{MeC}_{6} \mathrm{H}_{4}\right)_{3}, \mathrm{Sb}\left(o-\mathrm{MeC}_{6} \mathrm{H}_{4}\right)_{3}\right), \mathrm{em}$ combinação com dados de infravermelho, permitiram concluir que os complexos são quase sempre cis, apesar do significativo impedimento estérico devido aos cones de ângulo das estibinas, além de possibilitarem o estabelecimento das séries de influência cis e trans ${ }^{30}$. Estudos, também por Mössbauer, do núcleo ${ }^{129} \mathrm{I}$ de iodo-complexos, como no caso de compostos do tipo cis-[PtI $\left.\mathrm{L}_{2}\right]\left(\mathrm{L}=\mathrm{PPh}_{3}, \mathrm{AsPh}_{3}\right.$, $\mathrm{SbPh}_{3}$ ), entre outros, possibilitaram estabelecer séries de influência cis e trans para os ligantes empregados ${ }^{31}$.

Séries de compostos também foram especificamente preparadas para estudos de cinética, equilíbrio e/ou isomerização de ligação. Para esses propósitos, foram preparados complexos com outros ligantes, como $\left[\mathrm{PtCl}_{2}\left(\mathrm{PEt}_{3}\right) \mathrm{L}\right]\left(\mathrm{L}=\mathrm{PPh}_{3}, \mathrm{PMePh}_{2}, \mathrm{P}^{i} \mathrm{Pr}_{3}\right.$, $\left.\mathrm{P}\left(\mathrm{C}_{6} \mathrm{H}_{11}\right)_{3}, \mathrm{AsPh}_{3}, \mathrm{SbPh}_{3}\right)^{32}$. Todos os produtos obtidos apresentaram a conformação trans, exceto o composto com $\mathrm{L}=\mathrm{SbPh}_{3}$, que apresentou configuração cis. A suspensão em diclorometano do complexo trans- $\left[\mathrm{PtCl}_{2}\left(\mathrm{PEt}_{3}\right) \mathrm{L}\right]\left(\mathrm{L}=\mathrm{PPh}_{3}, \mathrm{PMePh}_{2}, \mathrm{AsPh}_{3}\right)$ tratada com L, produz sua isomerização a cis- $\left[\mathrm{PtCl}_{2}\left(\mathrm{PEt}_{3}\right) \mathrm{L}\right]$ com rendimentos acima de $95 \%$. A suspensão etanólica de cis- $\left[\mathrm{PtCl}_{2}\left(\mathrm{PEt}_{3}\right) \mathrm{L}\right]$ tratada com excesso de metil-lítio produz $\left[\mathrm{PtMe}_{2}\left(\mathrm{PEt}_{3}\right) \mathrm{L}\right]$; se $\mathrm{L}=$ $\mathrm{PPh}_{3}$, o produto é constituído por uma mistura eqüimolecular dos isômeros cis e trans e, se $\mathrm{L}=\mathrm{AsPh}_{3}$ ou $\mathrm{SbPh}_{3}$, o produto é exclusivamente o isômero $\mathrm{cis}^{32}$.

Pearson et ll. $^{33}$, em 1968, fizeram medidas de constantes de velocidade de segunda ordem para a reação de um grande número de nucleófilos, inclusive $\mathrm{SbPh}_{3}$, com iodometano e trans$\left.\left[\mathrm{PtCl}_{2} \text { (piridina) }\right)_{2}\right]$ em etanol. O produto da reação do complexo piridínico com $\mathrm{SbPh}_{3}$ não foi isolado.

Foram feitos estudos sobre a isomerização de ligação do íon tiocianato em complexos do tipo $\left[\mathrm{MX}_{2} \mathrm{~L}_{2}\right]\left(\mathrm{M}=\mathrm{Pd}, \mathrm{Pt}\right.$; $\mathrm{X}=\mathrm{SCN}^{-}$, NCS $;$ L = piridina, 2,2'-bipiridina, 4-n-amilpiridina, 1,10fenantrolina, tiouréia, etilenotiouréia, $\mathrm{PPh}_{3}, \mathrm{AsPh}_{3}$ e $\mathrm{SbPh}_{3}$ ), preparados a partir da reação de $\mathrm{K}_{2}\left[\mathrm{Pt}(\mathrm{SCN})_{4}\right]$ com o ligante apropriado em meio de etanol ${ }^{34}$. $\mathrm{Se} \mathrm{L}=\mathrm{SbPh}_{3}$, forma-se o tiocianatocomplexo $\left[\mathrm{Pt}(\mathrm{SCN})_{2}\left(\mathrm{SbPh}_{3}\right)_{2}\right]$; se $\mathrm{L}=\mathrm{PPh}_{3}$, é obtido o isotiocianatocomplexo $\left[\mathrm{Pt}(\mathrm{NCS})_{2}\left(\mathrm{PPh}_{3}\right)_{2}\right]$. A explicação para a presença de diferentes modos de coordenação do íon tiocianato é dada em função de fatores eletrônicos e estéricos, os quais podem alterar a natureza da ligação metal-ligante ${ }^{34}$.

A carbonilação de compostos do tipo $\left[\mathrm{PtX}(\mathrm{R}) \mathrm{L}_{2}\right](\mathrm{X}=\mathrm{Cl}, \mathrm{Br}$, $\mathrm{I} ; \mathrm{R}=\mathrm{Ph}, p-\mathrm{MeOC}_{6} \mathrm{H}_{4}, p-\mathrm{CF}_{3} \mathrm{C}_{6} \mathrm{H}_{4}, p-\mathrm{O}_{2} \mathrm{NC}_{6} \mathrm{H}_{4}, \mathrm{PhCH}_{2}-, \mathrm{Me} ; \mathrm{L}=$ $\mathrm{PPh}_{3}, \mathrm{P}\left(\mathrm{C}_{6} \mathrm{H}_{11}\right)_{3}, \mathrm{P}(\mathrm{OPh})_{3}, \mathrm{P}\left(p-\mathrm{CF}_{3} \mathrm{C}_{6} \mathrm{H}_{4}\right)_{3}, \mathrm{P}\left(p-\mathrm{Me}_{2} \mathrm{NC}_{6} \mathrm{H}_{4}\right)_{3}, \mathrm{P}(p-$ $\left.\left.\mathrm{MeOC}_{6} \mathrm{H}_{4}\right)_{3}, \mathrm{P}\left(p-\mathrm{MeC}_{6} \mathrm{H}_{4}\right)_{3}\right)$ produzindo $\left[\mathrm{PtX}(\mathrm{COPh}) \mathrm{L}_{2}\right]$ foi estudada por Garrou e Heck, em 1976, e os dados cinéticos para absorção de monóxido de carbono indicaram que, para os complexos com $\mathrm{AsPh}_{3}$ e $\mathrm{SbPh}_{3}$, a velocidade de absorção é cerca de 300 vezes maior que para o correspondente complexo com $\mathrm{PPh}_{3}{ }^{35}$.

Foram preparados hidreto-complexos do tipo $\left[\mathrm{Pt}(\mathrm{H})\left\{\mathrm{P}\left(\mathrm{C}_{6} \mathrm{H}_{11}\right)_{3}\right\}_{2} \mathrm{~L}\right]\left[\mathrm{PF}_{6}\right] \quad\left(\mathrm{L}=\mathrm{PEt}_{3}, \mathrm{PPh}_{2} \mathrm{Me}, \quad \mathrm{PPhMe}_{2}\right.$, $\mathrm{PPh}_{2}\left(\mathrm{C}_{6} \mathrm{H}_{11}\right), \mathrm{PPh}\left(\mathrm{C}_{6} \mathrm{H}_{11}\right)_{2}, \mathrm{P}\left(\mathrm{C}_{6} \mathrm{H}_{11}\right)_{3}, \mathrm{P}^{i} \mathrm{Pr}_{3}, \mathrm{PBu}_{3}, \mathrm{P}\left(p-\mathrm{MeC}_{6} \mathrm{H}_{4}\right)_{3}$, $\left.\mathrm{P}\left(m-\mathrm{MeC}_{6} \mathrm{H}_{4}\right)_{3}, \mathrm{P}\left(o-\mathrm{MeC}_{6} \mathrm{H}_{4}\right)_{3}, \mathrm{AsPh}_{3}, \mathrm{SbPh}_{3}\right)$ por reação de $\mathrm{L}$ com $\left[\mathrm{Pt}(\mathrm{H})\left\{\mathrm{P}\left(\mathrm{C}_{6} \mathrm{H}_{11}\right)_{3}\right\}_{2}(\right.$ solvente $\left.)\right]\left[\mathrm{PF}_{6}\right]$ (solvente $\left.=\mathrm{Me}_{2} \mathrm{O}, \mathrm{MeOH}\right)$ para estudos de efeitos estéricos na química de hidretos de platina bivalente ${ }^{36}$.
A estrutura cristalina do complexo $\left[\mathrm{PtCl}_{2}(\mathrm{tmcb})\left(\mathrm{SbPh}_{3}\right)\right]$ (tmcb $=$ tetrametilciclobutadieno) foi descrita por Heinemann e colaboradores $^{37}$, em 1996. Logo em seguida, Wendt e Elding ${ }^{38}$ descreveram as estruturas cristalinas de cis- $\left[\mathrm{PtCl}_{2}\left(\mathrm{SbPh}_{3}\right)_{2}\right]$ e trans$\left[\mathrm{PtI}_{2}\left(\mathrm{SbPh}_{3}\right)_{2}\right]$, demonstrando que a influência trans de $\mathrm{SbPh}_{3}$ é comparável àquela de $\mathrm{PPh}_{3}$, contrariando o que vinha sendo descrito na literatura. Eles também descreveram o efeito trans e a influência trans de $\mathrm{SbPh}_{3}$ e $\mathrm{PPh}_{3}$ em complexos $\left[\mathrm{PtI}_{3}\left(\mathrm{EPh}_{3}\right)\right]^{-}(\mathrm{E}=\mathrm{P}$, $\mathrm{Sb})$ com piridina e piridinas substituídas. O maior efeito trans observado é explicado pelo fato de $\mathrm{SbPh}_{3}$ ser um doador $\sigma$ mais fraco que $\mathrm{PPh}_{3}$, enquanto o maior efeito trans é devido ao fato de $\mathrm{SbPh}_{3}$ ser um melhor receptor $\pi$ que $\mathrm{PPh}_{3}$.

Sharma e colaboradores ${ }^{39}$, em 2000 , descreveram reações de dibromodifenilantimoniatos(III) com $\mathrm{PtCl}_{2}$ e cis-[ $\left.\mathrm{PtCl}_{2}\left(\mathrm{PPh}_{3}\right)_{2}\right]$, produzindo complexos de platina(II) contendo $\mathrm{SbPh}_{3}$. A formação dos complexos cis- $\left[\mathrm{PtBr}_{2}\left(\mathrm{SbPh}_{3}\right)_{2}\right]$, trans-[PtBr$\left.(\mathrm{Ph})\left(\mathrm{SbPh}_{3}\right)_{2}\right]$, $\left[\mathrm{NMe}_{4}\right]\left[\mathrm{PtBr}_{3}\left(\mathrm{SbPh}_{3}\right)\right]$ e cis- $\left[\mathrm{PtBr}_{2}\left(\mathrm{PPh}_{3}\right)\left(\mathrm{SbPh}_{3}\right)\right]$, para os quais foram obtidas as estruturas cristalinas, é explicada em termos de um possível mecanismo de migração de um grupo fenila.

Henderson et al..$^{40}$, em 2003, estudando isomerismos de coordenação em produtos de reação de $\mathrm{K}_{2}\left[\mathrm{PtCl}_{4}\right]$, ácido salicilidroxâmico e excesso de $\mathrm{Me}_{3} \mathrm{~N}$ com $\mathrm{EPh}_{3}(\mathrm{E}=\mathrm{As}, \mathrm{Sb})$, verificaram que as estruturas dos complexos obtidos são dependentes de E, como nos compostos indicados a seguir:

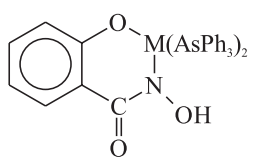<smiles>Oc1ccccc1C1=NO[AsH2]O1</smiles>

\section{COMPLEXOS DE PLATINA(0) COM TRIFENILESTIBINA}

Para compostos zerovalentes de platina com trifenilestibina, a primeira referência encontrada na literatura é de Takahashi e colaboradores ${ }^{41}$, de 1966. Estudando o comportamento catalítico de fosfinas de paládio e platina em reações de oxigenação, foi obtido o complexo $\left[\mathrm{Pt}\left(\mathrm{PPh}_{3}\right)_{3}\left(\mathrm{SbPh}_{3}\right)\right]$ a partir da reação de $\left[\mathrm{Pt}\left(\mathrm{PPh}_{3}\right)_{4}\right]$ com $\mathrm{SbPh}_{3}$. A partir de $\left[\mathrm{Pd}\left(\mathrm{PPh}_{3}\right)_{4}\right]$, em reação similar, foi obtido o complexo $\left[\mathrm{Pd}\left(\mathrm{PPh}_{3}\right)_{2}\left(\mathrm{SbPh}_{3}\right)_{2}\right]$.

Garrou e Hartwell ${ }^{42}$, em 1976, ao estudarem compostos zerovalentes de níquel, paládio e platina com triarilestibinas, prepara-

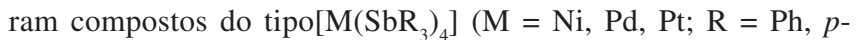
$\mathrm{MeC}_{6} \mathrm{H}_{4}, p$ - $\mathrm{MeOC}_{6} \mathrm{H}_{4}$ ) e os complexos com ligantes mistos $\left[\mathrm{M}\left(\mathrm{PPh}_{3}\right)_{2}\left(\mathrm{SbR}_{3}\right)_{2}\right]\left(\mathrm{M}=\mathrm{Pd}, \mathrm{Pt} ; \mathrm{R}=\mathrm{Ph}, p-\mathrm{MeC}_{6} \mathrm{H}_{4}\right)^{41}$. No entanto, falharam todas as tentativas que fizeram para obtenção de $\left[\mathrm{Pt}\left(\mathrm{SbPh}_{3}\right)_{4}\right]$.

Posteriormente, Scherer e colaboradores ${ }^{43}$, em 1982, prepararam complexos do tipo $\left[\mathrm{PtL}_{3}\right]\left(\mathrm{L}=\mathrm{P}\left(: \mathrm{NCMe}_{3}\right) \mathrm{N}\left(\mathrm{SiMe}_{3}\right)\left(\mathrm{CMe}_{3}\right)\right)$ a partir de $\left[\mathrm{Pt}(\operatorname{cod})_{2}\right](\operatorname{cod}=1,5$-ciclooctadieno $)$. O tratamento de um mol de $\left[\mathrm{PtL}_{3}\right]$ com um mol de $\mathrm{ER}_{3}(\mathrm{E}=\mathrm{As}, \mathrm{Sb} ; \mathrm{R}=\mathrm{Me}, \mathrm{Ph}$, $\mathrm{OMe}, \mathrm{OPh})$ produz $\left[\mathrm{PtL}_{2}\left(\mathrm{ER}_{3}\right)\right] \mathrm{e}$, com dois mols, produz $\left[\mathrm{PtL}\left(\mathrm{ER}_{3}\right)_{2}\right]$.

Na revisão até aqui apresentada, chama atenção que o interesse em preparar complexos de platina com $\mathrm{SbPh}_{3}$ se deva à complementação de séries para fins de estudos condutimétricos, espectroscópicos e cinéticos, entre outros. Somente nos trabalhos de McAuliffe et ll $^{25}$, de 1977 , e de Garrou e Hartwell ${ }^{42}$, de 1976, é que se observa a preocupação exclusiva de preparar complexos de platina bivalente e zerovalente contendo $\mathrm{SbPh}_{3}$.

Cabe ressaltar a tentativa frustrada de preparação de um composto binário de $\mathrm{SbPh}_{3}$ e $\mathrm{Pt}(0)$, que resultou em um precipitado negro, identificado como platina metálica ${ }^{42}$. 
Na tentativa de preparação de $\left[\mathrm{Pt}\left(\mathrm{SbPh}_{3}\right)_{4}\right]$, Barbiéri et al. ${ }^{44}$, em 1987, reagiram $\left[\mathrm{PtCl}_{2}\left(\mathrm{SbPh}_{3}\right)_{2}\right]$ com excesso de $\mathrm{SbPh}_{3}$, em atmosfera de argônio e com adição de $\mathrm{NaBH}_{4}$, obtendo com alto rendimento um complexo formalmente com 16 elétrons, formulado como $\left[\mathrm{Pt}\left(\mathrm{SbPh}_{3}\right)_{3}\right]^{44}$. Verificou-se que esse composto, pode ser preparado sem cuidados de atmosfera especial, sendo excepcionalmente estável ao ar, o que foi atribuído ao cone de ângulo maior do ligante $\mathrm{SbPh}_{3}$ em relação à $\mathrm{AsPh}_{3}$ e $\mathrm{PPh}_{3}$.

No entanto, quando a tentativa de síntese de $\left[\mathrm{Pt}\left(\mathrm{SbPh}_{3}\right)_{3}\right]$ foi conduzida sob atmosfera de nitrogênio à pressão ordinária, obteve-se o complexo $\left[\left\{\mathrm{Pt}\left(\mathrm{SbPh}_{3}\right)_{2}\right\}_{2} \mathrm{~N}_{2}\right]$, o primeiro exemplo de um dinitrogênio-complexo de platina, com o dinitrogênio em ponte, estável à temperatura ambiente ${ }^{44}$. Sob atmosfera de acetileno, foi preparado o complexo $\left[\left\{\mathrm{Pt}\left(\mathrm{SbPh}_{3}\right)_{2}\right\}_{2} \mathrm{C}_{2}\right]$, com uma ponte de acetileno; enquanto que sob atmosfera de monóxido de carbono, foi preparado o composto $\left[\mathrm{Pt}(\mathrm{CO})_{2}\left(\mathrm{SbPh}_{3}\right)_{2}\right]$.

Klein et $a l .{ }^{45}$, em 1990, realizaram estudos de aniquilação de positrônios em complexos de metais da tríade do níquel, incluindo $\left[\mathrm{Pt}\left(\mathrm{SbPh}_{3}\right)\right]$, verificando que a probabilidade de formação e o tempo de vida do orto-positrônio são dependentes do estado de oxidação dos metais e das geometrias dos compostos.

Barbiéri et al. ${ }^{46}$, em 1996, estudaram os compostos $\left[\mathrm{PtCl}_{2}\left(\mathrm{SbPh}_{3}\right)_{2}\right],\left[\mathrm{Pt}\left(\mathrm{SbPh}_{3}\right)_{3}\right],\left[\left\{\mathrm{Pt}\left(\mathrm{SbPh}_{3}\right)_{2}\right\}_{2} \mathrm{~N}_{2}\right],\left[\left\{\mathrm{Pt}\left(\mathrm{SbPh}_{3}\right)_{2}\right\}_{2} \mathrm{C}_{2}\right]$ e $\left[\mathrm{Pt}(\mathrm{CO})\left(\mathrm{SbPh}_{3}\right)_{2}\right]$ por meio de análises termogravimétrica e térmica diferencial, indicando que o produto final de termodecomposição é uma liga platina-antimônio, cuja composição é dependente da proporção estequiométrica dos metais nos complexos.

Estudando a reatividade dos complexos $\left[\left\{\mathrm{Pt}\left(\mathrm{SbPh}_{3}\right)_{2}\right\}_{2} \mathrm{~N}_{2}\right]$, $\left[\left\{\mathrm{Pt}\left(\mathrm{SbPh}_{3}\right)_{2}\right\}_{2} \mathrm{C}_{2}\right]$, Barbiéri et al. ${ }^{47}$, em 2005, verificaram que eles se convertem espontaneamente em $\left[\mathrm{Pt}\left(\mathrm{SbPh}_{3}\right)_{3}\right]$ no prazo de alguns dias, processo que é acelerado pela suspensão dos complexos em etanol ou benzeno ou por aquecimento até $100{ }^{\circ} \mathrm{C}$ durante $1 \mathrm{~h}$. A reação com $\mathrm{HBr}$, em meio de etanol ou benzeno, produz $\left[\mathrm{PtBr}_{2}\left(\mathrm{SbPh}_{3}\right)_{2}\right]$, enquanto que a reação com $\mathrm{Br}_{2}$, nas mesmas condições, produz um composto dimérico $\left[\mathrm{PtBr}_{3}\left(\mathrm{SbPh}_{3}\right)_{2}\right]_{2}$, contendo pontes de bromo ${ }^{47}$.

\section{CONCLUSÃO}

Mesmo considerando que a maior parte dos trabalhos indicados na presente revisão tenha sido publicada nos anos 70 e 80 do século passado, fica demonstrado que a química dos compostos de platina com trifenilestibina $\left(\mathrm{SbPh}_{3}\right)$ não foi alvo, ainda, de estudos específicos e aprofundados. Ainda é importante considerar o fato de que nos estudos de compostos com $\mathrm{ER}_{3}(\mathrm{E}=\mathrm{P}, \mathrm{As} ; \mathrm{R}=\mathrm{Ph})$ como ligantes ancilares no átomo central de platina, estes ligantes podem conter outros grupos $\mathrm{R}$ alquila ou arila ou ser do tipo $\mathrm{ER}_{2} \mathrm{R}$ ', enquanto que para $\mathrm{E}=\mathrm{Sb}$, $\mathrm{R}$ é sempre o grupo fenila.

As observações de comportamentos físico-químicos diferenciados para os compostos de platina-trifenilestibina, quando comparados aos representantes das séries contendo níquel ou paládio, têm sido motivadoras para os autores retomarem os estudos destas substâncias.

\section{REFERÊNCIAS}

1. Jensen, K. A.; Z. Anorg. Allgem. Chem. 1936, 229, 225

2. Beck, W.; Scharpp, K.; Oether, C.; Chem. Ber. 1974, 107, 1380.

3. Norbury, A. H.; Pinha, A. I. P.; J. Chem. Soc. (A) 1968, 1598.

4. Westland, A. D.; J. Chem. Soc. (A) 1965, 3060.

5. Eaborn, C.; Kundu, K.; Pidcok, A.; J. Chem. Soc., Dalton Trans. 1981, 933.

6. Hulley, G.; Johnson, F. G.; Lewis, G.; J. Chem. Soc. (A) 1970, 1732.

7. Clark, H. C.; Goel, A. B.; Goel, R. G.; Goel, S.; Inorg. Chem. 1980, 19, 3220 .
8. Goel, A. B.; Goel, S.; Angew. Chem., Int. Ed. 1984, 23, 375.

9. Goel, A. B.; Goel, S.; Inorg. Chim. Acta 1984, 82, 41.

10. Cherwinski, W. J.; Clark, H. C.; Can. J. Chem. 1969, 47, 2665.

11. Usón, R.; Forniés, J.; Espinet, R.; Navarro, R.; Martinez, F.; Tomás, M.; J. Chem. Soc., Chem. Commun. 1977, 789; Usón, R.; Forniés, J.; Martínez, F.; Tomás, M.; J. Chem. Soc., Dalton Trans. 1980, 888; Usón, R.; Forniés, J.; Martínez, F.; Tomás, M.; Reoyo, I.; Organometallics 1983, 2, 1386; Usón, R.; Forniés, J.; Menjón, B.; Cotton, F. A.; Inorg. Chem. 1985, 24, 4651 .

12. Saez, I. M.; Lopez, G.; Garcia, G.; Anales de la Universidad de Murcia. Ciencias, 1976-1977, 35, 157. (CA 96:85723t).

13. Eaborn, C.; Pidcock, A.; Steele, B. R.; J. Chem. Soc., Dalton Trans. 1976, 767; Eaborn, C.; Odell, K. J.; Pidcock, A.; J. Chem. Soc., Dalton Trans. 1978, 1288.

14. Toniolo, L.; Giustiniani, M.; Belluco, U.; J. Chem. Soc. (A) 1969, 2666.

15. Miyamoto, T.; Sasaky, Y. A.; Chem. Lett. 1976, 191.

16. Kato, M.; Urabe, H.; Osawa, Y.; Saito, T.; Sasaki, Y.; J. Organomet. Chem. 1976, 121, 81 .

17. Alinson, J. M. C.; Stephenson, T. A.; Gould, R. O.; J. Chem. Soc. (A) 1971, 3690 .

18. Kistner, C. R.; Blackman, J. P.; Harris, W. C.; Inorg. Chem. 1969, 8, 2165.

19. Clark, H. C.; Manzer, L. E.; J. Organomet. Chem. 1973, 59, 441.

20. Payne, N. C.; Stephan, D. W.; J. Organomet. Chem. 1981, 221, 203.

21. Beck, W.; Holsboer, F.; Z. Naturforsch., B: Chem. Sci. 1973, 28, 511. (CA $81: 8156 y)$.

22. Grutsch, P. A.; Zeller, W. V.; Fehlner, T. P.; Inorg. Chem. 1973, 12, 1431.

23. Gavrilova, I. V.; Deposited Doc. 1980 (CA 97:84075s); Gavrilova, I. V.; Gel'Fman, M. I.; Ivannikova, N. V.; Kiseleva, N. V.; Razumovskii, V. V.; Zh. Neorg, Khim. 1973, 18, 194. (CA 89202y); Gavrilova, I. V.; Gel'Fman, M. I.; Ivannikova, N. V.; Razumovskii, V. V.; Zh. Neorg. Khim. 1971, 16, 1124. (CA 74:150588g).

24. Janos, M.; Goggin, P. L.; Kem. Kozl. 1984, 61, 275. (CA 102:211900f).

25. McAuliffe, C. A.; Niven, I. E.; Parish, R. V.; Inorg. Chim. Acta 1977, 22, 239.

26. Appleton, T. G.; Chisholm, M. H.; Clark, H. C.; Manzer, L. E.; Inorg. Chem. 1972, 11, 1786.

27. Appleton, T. G.; Chisholm, M. H.; Clark, H. C.; Manzer, L. E.; Can. J. Chem. 1973, 51, 2243.

28. Motschi, H.; Pregosin, P. S.; Inorg. Chim. Acta 1980, 40, 141.

29. Appleton, T. G.; Bennett, M. A.; Inorg. Chem. 1978, 17, 738.

30. McAuliffe, C. A.; Niven, I. E.; Parish, R. V.; J. Chem. Soc., Dalton Trans. 1977, 1901.

31. Dale, B. W., Dickinson, R. J.; Parish, R. V.; Chem Phys. Lett. 1974, 24, 286; Dickinson, R. J.; Parish, R. V.; Dale, B. W.; J. Chem. Soc., Dalton Trans. 1980, 895.

32. Clark, H. C.; Goel, A. B.; Wong, C. S.; J. Organomet. Chem. 1980, 190, C101.

33. Pearson, R. G.; Sobel, H.; Songstad, J.; J. Am. Chem. Soc. 1968, 90, 319.

34. Basolo, F.; Baddley, W. H.; Burmeister, J. L.; Inorg. Chem. 1964, 3, 1202; Burmeister, J. L.; Basolo, F.; Inorg. Chem. 1964, 3, 1587.

35. Garrou, P. E.; Heck, R. F.; J. Am. Chem. Soc. 1976, 98, 4115.

36. Clark, H. C.; Dymarski, M. J.; Oliver, J. D.; J. Organomet. Chem. 1978, $154, \mathrm{C} 40$.

37. Heinemann, F. W.; Gerisch, M.; Schenzel, K.; Steinborn, D.; Z. Kristallogr. 1996, 221, 388.

38. Wendt, O. F.; Doctoral thesis, University of Messina, Italy, 1997; Wendt, O. F.; Elding, L. I.; J. Chem. Soc., Dalton Trans. 1997, 4725; Wendt, O. F.; Scodinu, A.; Elding, L. I.; Inorg. Chim. Acta 1998, 277, 237.

39. Sharma, P.; Cabrera, A.; Sharma, M.; Alvarez, C.; Arias, J. L.; Gomez, R. M.; Hernandez, S.; Z. Anorg. Allg. Chem. 2000, 626, 2330.

40. Henderson, W.; Evans, C.; Nocholson, B. K.; Fawcett, J.; Dalton Trans. 2005, 13, 2691.

41. Takahashi, S.; Sonogashira, K.; Hagihara, N.; Nippon Kagaku Zasshi 1966, 87, 610. (CA 65:14485d).

42. Garrou, P. E.; Hartwell, G. E.; Inorg. Chem. 1976, 15, 730.

43. Scherer, O. J.; Konrad, R.; Krueger, C.; Tray, Y. H.; Chem. Ber. 1982, 115, 414.

44. Barbiéri, R. S.; Klein, S. I.; Massabni, A. C.; J. Chem. Soc., Chem. Commun. 1987, 1617; Barbiéri, R. S.; Tese de Doutorado, Universidade de São Paulo, Brasil, 1989.

45. Klein, S. I.; Barbiéri, R. S.; Marques Netto, A.; Silva, M. E. S. R.; Machado, J. C.; J. Braz. Chem. Soc. 1990, 1, 81 .

46. Barbiéri, R. S.; Bellato, C. R.; Massabni, A. C.; Termochim. Acta 1995, 259, 277.

47. Barbiéri, R. S.; Bellato, C. R.; Massabni, A. C.; Transition Met. Chem. 2005, 30, 217. 